\title{
Efecto de los ambientes de aprendizaje en estudiantes de manejo de la salud y bienestar animal de la licenciatura de medicina veterinaria en la UdeG
}

\section{Effect of learning environments on students of animal health and welfare management of the degree of veterinary medicine at the UdeG}

\author{
MEDINA-LERENA, Miriam Susana *†, COLÍN-MARTINEZ, Marco Antonio, RIMOLDI RENTERÍA, \\ Ma. De Jesús y PÉREZ-RAMÍREZ, Miguel Ángel
}

Centro Universitario de Ciencias Biológicas y Agropecuarias (CUCBA), Universidad de Guadalajara, Camino Ing. Ramón Padilla Sánchez No. 2100, Predio Las Agujas, Nextipac, Zapopan, Jalisco, México. C. P. 45510.

\author{
ID $1^{\text {er }}$ Autor: Miriam Susana, Medina-Lerena / ORC ID: 0000-0003-4358-5400 \\ ID $1^{\text {er }}$ Coautor: Marco Antonio, Martínez Colín / ORC ID: 0000-0003-4905-0386 \\ ID $2^{\text {do }}$ Coautor: Ma. De Jesús, Rimoldi-Rentería / ORC ID: 0000-0002-8432-1068 \\ ID $3^{\text {er }}$ Coautor: Miguel Ángel, Pérez-Ramírez / ORC ID: 0000-0002-5393-8751
}

DOI: $10.35429 / \mathrm{JCP} .2019 .9 .3 .29 .35$

Recibido 03 de Junio, 2019; Aceptado 30 de Septiembre, 2019

\section{Resumen}

Los docentes buscan modelos adecuados que proporcionen a estudiantes universitarios las condiciones y elementos esenciales que estimulen el desarrollo de habilidades y competencias para toda la vida. El concepto de ambientes de aprendizajes, no solo es hablar de infraestructura, materiales o recursos de apoyo, que de cierto modo son importantes, pero que, en sí, la esencia de este dependerá de la iniciativa, creatividad, capacidad e interacción de la persona que esté al frente del proceso enseñanza-aprendizaje que es el facilitador. El objetivo fue conocer el ambiente por el cual el alumno aprende mejor como parte de la apropiación de saberes y construcción del conocimiento. Se evaluó un grupo de estudiantes $(\mathrm{n}=40)$, del Curso de Manejo de la Salud y Bienestar Animal de la Licenciatura de Veterinaria, mediante un diseño descriptivo por medio de ambientes virtuales (T1), dentro del aula (T2) y ambientes reales (T3). Resultando el mejor ambiente el T3, seguido de T1. La finalidad de los ambientes de aprendizaje es proporcionar recursos y desarrollar las condiciones que contribuyan y faciliten la adquisición del conocimiento. Por otro lado el proceso de aprendizaje bien empleados contribuyen a la adquisición de aprendizajes proporcionando a su vez actitudes y valores en el alumno.

Ambientes de aprendizaje, Aprendizaje virtual, Aprendizaje real

\begin{abstract}
Teachers look for suitable models that provide university students with the conditions and essential elements that stimulate the development of skills and competences for a lifetime. The concept of learning environments is not only to talk about infrastructure, materials or support resources, which in some way are important, but that in itself, the essence of this will depend on the initiative, creativity, capacity and interaction of the person who be at the forefront of the teaching-learning process that is the facilitator. The objective was to know the environment through which the student learns better as part of the appropriation of knowledge and knowledge construction. A group of student $(n=40)$, of the course of Management of Animal Health and Welfare of the Veterinary Degree, was evaluated through a descriptive design through virtual environments (T1), within the classroom (2) and real environments (T3). The best environment being T3, followed by $\mathrm{T} 1$. The purpose of learning environments is to provide resources and develop conditions that contribute and facilitate the acquisition of knowledge. On the other hand, the learning processes well used contribute to the acquisition of learning while providing attitudes and values in the student.
\end{abstract}

Learning environment, Virtual learning, Real learning

Citación: MEDINA-LERENA, Miriam Susana, COLÍN-MARTINEZ, Marco Antonio, RIMOLDI RENTERÍA, Ma. De Jesús y PÉREZ-RAMÍREZ, Miguel Ángel. Efecto de los ambientes de aprendizaje en estudiantes de manejo de la salud y bienestar animal de la licenciatura de medicina veterinaria en la UdeG. Revista de Pedagogía Crítica. 2019, 3-9: 29-35

\footnotetext{
* Correspondencia al Autor (Correo electrónico: miriam.mlerena@academicos.udg.mx)

$\dagger$ Investigador contribuyendo como primer autor.
} 


\section{Introducción}

Los ambientes de aprendizaje no solamente abarcan métodos de enseñanza sino también de las oportunidades que tienen los estudiantes para aprender y adquirir conocimientos. Asimismo, el diseño de los ambientes depende, en gran medida, de los modelos de actuación docente y los métodos de enseñanza educativa acordes a las características de los estudiantes (Navaridas y Jiménez, 2016). Actualmente la educación ha sido fuertemente influenciada por las Tecnologías de la Información y de la Comunicación que desafían y producen un cambio en la manera de concebir la vida ante un mundo en constante desarrollo. Los estudiantes utilizan las aplicaciones de enseñanza y pueden ser usadas como complemento en la manera de aprender (Saraiva et. al., 2006).

Un docente como facilitador y mediador del proceso de enseñanza debe de llevar a cabo la implementación de didácticas con ambientes $\mathrm{y}$ estrategias de aprendizaje que sean innovadoras y creativas. También pueden proporcionar al estudiante elementos que ayuden a facilitar, motivar y comprender el conocimiento para que con ello sea capaz de trasmitir y aplicar en su enseñanza a través de su estudio durante la etapa estudiantil en la Universidad. De esta manera logrará adquirir competencias, conocimientos y habilidades. Mismas que les servirán para la formación personal y profesional que los lleven a incluirse en un mundo que exige mejores elementos profesionales y competitivos (Moreno, 2005).

Las clases presenciales y/o no presenciales, son escenarios donde se cuenta con flexibilidad, estos tienen la ventaja de acceder a la información y al mismo tiempo se puede compartir, debatir e intercambiar información o simplemente pueden interactuar en el desarrollo de las actividades programadas.

\section{Justificación}

Los ambientes de aprendizaje deben proporcionar a los estudiantes, elementos esenciales, que propicien una enseñanza que estimule el desarrollo de sus habilidades, competencias y condiciones necesarias que permiten problematizar, descubrir, comprender, motivar y/o asimilar situaciones o contenidos educativos de la vida diaria desde su propia perspectiva (Rodríguez, 2014).
Por lo que el docente debe de buscar fomentar el uso de herramientas necesarias y al mismo tiempo escenarios indispensables para que el estudiante adquiera los conocimientos esenciales y de interés hacia su propio aprendizaje. Dado que los ambientes ayudan al dominio de competencias con recursos que estén disponibles, mismos que estén actualizados y en vanguardia para competir por mejores oportunidades profesionales en el campo laboral. Los contextos de enseñanza-aprendizaje han cambiado con la presencia de las aplicaciones de tecnologías en la educación; su uso se ha convertido en una herramienta casi indispensable para la formación de los estudiantes.

De aquí que se buscó y analizó si los ambientes de aprendizaje utilizados en la competencia de Manejo de la Salud y Bienestar Animal proporcionan elementos que apoyan el desarrollo de habilidades y competencias en el estudiante de Medicina Veterinaria. Es importante poder reconocer el mejor ambiente de aprendizaje donde el alumno se apropie de conocimientos a partir de la información recibida. Misma que apoye en su formación profesional y en la solución de problemas presentados en su vida diaria. De ahí que es indispensable conocer si los ambientes de aprendizaje que se utilizan son los mejores para que el estudiante cree su propio conocimiento con el objetivo de que obtenga un aprendizaje significativo en la competencia.

\section{Planteamiento del problema}

Uno de los objetivos más importantes de la educación es que el alumno sea autodidacta y resuelva problemas cotidianos y reales en su entorno. Las competencias forman parte del proceso evolutivo al momento de aprender, siendo más reflexivo, crítico y al mismo tiempo competente en cualquier área en la que se dedique profesionalmente. Los ambientes de aprendizaje permiten las interacciones de manera constante entre alumno-profesor, alumno-alumno, alumno-herramientas.

El éxito de estos ambientes de aprendizaje se debe al papel dinámico que el docente establece cuando interactúa con sus estudiantes logrando así una asistencia diaria a clase y puedan lograr un proceso de enseñanza aprendizaje, siendo el principal objetivo de la educación (Rubí et. al., 2017). 


\section{Hipótesis}

Los ambientes de aprendizaje utilizados en un curso de Manejo de la Salud y Bienestar Animal evidenciarán el efecto sobre el estudiante para obtener un aprendizaje significativo.

\section{Objetivo general}

Evaluar el efecto de los ambientes de aprendizaje utilizadas en Estudiantes de la competencia de Manejo de la Salud Animal de la Licenciatura de Medicina Veterinaria en la UdeG.

\section{Objetivos particulares}

- Determinar si los ambientes causan un efecto en el aprendizaje de los alumnos.

- Evaluar el auto-aprendizaje mediante estrategias utilizadas en el curso.

Evaluar el desempeño adquirido en un curso de Manejo y Bienestar Animal.

Evaluar las actividades dentro y fuera del aula como estrategia de aprendizaje.

Determinar si los ambientes y las estrategias usadas provocan un efecto en el aprendizaje significativo en el estudiante.

\section{Marco teórico}

En las últimas décadas ha tomado gran interés el desarrollo de la psicología cognitiva con sus aportes en el campo educativo, básicamente a través del aprendizaje significativo y el aprendizaje estratégico, nuevos planteamientos sobre la inteligencia humana que la conciben como múltiples, diversas y con cambios en los procesos de formación, en los distintos niveles educativos (Villamizar, 2008).

Navaridas y Jiménez (2016) investigaron los conceptos que tienen los estudiantes universitarios acerca de la utilidad y/o eficacia de los ambientes de aprendizaje que son utilizados por los docentes en sus actividades diarias. Los resultados indicaron que el estudio individual y las explicaciones del profesor siguen siendo los ambientes más valorados por los estudiantes en términos de eficacia para el logro de los objetivos de aprendizaje (Navaridas y Jiménez, 2016).
Los resultados en alumnos evaluados antes y después del uso e integración de la tecnología en su desarrollo han aumentado no solo en conocimientos específicos, sino también en la ortografía y el aprendizaje significativo (Márquez y Prats, 2011).

Los ambientes de aprendizaje virtuales pueden ser utilizados como complemento en clases presenciales y/o no presenciales. Los cuales tienen la flexibilidad y la ventaja que al momento de acceder a la información ayudan a compartir, debatir e intercambiar información o simplemente pueden interactuar en equipos por medio el desarrollo de las actividades programadas en el curso. El diseño de multimedia interactiva, permite ser utilizada como una herramienta tecnológica de apoyo didáctico para los docentes con el propósito de mejorar el aprendizaje, lo cual permite enseñar con mayor facilidad, agradable, llamativa y a la vez dinámica. Logrando que los estudiantes muestren interés por adquirir el nuevo aprendizaje (Pantoja, 2017).

\section{Material y métodos}

El presente estudio se realizó con un grupo de la Competencia de Manejo de la Salud y Bienestar Animal de la Licenciatura de Medicina Veterinaria y Zootecnia de la Universidad de Guadalajara. El grupo se conformó por una población de 40 alumnos inscritos durante el ciclo 2019-A. Con edades de los 18 a los 38 años, hombres y mujeres. Mediante un diseño descriptivo, con el propósito de buscar y conocer cuál es el mejor ambiente de aprendizaje. En el cual se lleva a cabo el proceso de enseñanzaaprendizaje al momento de adquirir sus conocimientos, a partir de la información recibida durante el Curso. Esto es complementado con un proyecto integrador en el cual son aplicadas todas las competencias adquiridas durante el ciclo. Se aplicaron tres tratamientos diferentes a los estudiantes en los cuales se menciona brevemente una descripción de cada uno y a su vez la estrategia didáctica empleada, como sigue a continuación:

$\mathrm{T} 1=$ (Por medio de ambientes virtuales) Los estudiantes trabajaron en un aula fuera con ambientes virtuales con el fin de buscar información para un proyecto integrador, el cual se realizó con un equipo de 5 personas. Se realizaron tareas tanto individuales y grupales. Posteriormente se realizó la evaluación terminada la unidad de competencia.

MEDINA-LERENA, Miriam Susana, COLÍN-MARTINEZ, Marco Antonio, RIMOLDI RENTERÍA, Ma. De Jesús y PÉREZ-RAMÍREZ, Miguel Ángel. Efecto de los ambientes de aprendizaje en estudiantes de manejo de la salud y bienestar animal de la licenciatura de medicina veterinaria en la UdeG. Revista de Pedagogía Crítica. 2019 
$\mathrm{T} 2=($ Dentro del aula $)$ Los estudiantes trabajaron con la información recibida haciendo las actividades en clase con tareas de la unidad de competencia y posteriormente se realizó la evaluación.

$\mathrm{T} 3=($ En ambientes reales $)$ Los estudiantes trabajaron con la información de la competencia para revisar un caso y realizar un diagnóstico. Dichas actividades fueron realizadas en campo. Una vez terminando el diagnóstico se realizó una evaluación.

Para las actividades virtuales, se solicitó una sala de biblioteca con el objetivo de explicar la forma de buscar y obtener información confiable desde la biblioteca digital. Se les enseño como recuperar los recursos para elaborar un proyecto integrador, el cual es un trabajo de investigación en el cual se les asigna un tema y lo desarrollan. Dicho proyecto debería ser escrito y elaborado con fuentes confiables y citadas en el estilo APA. En la sala solicitada se asignó un equipo de cómputo a cada uno de los estudiantes con el fin de que cada uno tuviera una computadora para trabajar de manera individual, sin embargo, no se contaban con los suficientes equipos por lo que se tuvo que compartir algunos equipos para dos personas.

Los proyectos fueron evaluados por medio de rubricas y al final se realizó un cartel en el cual incluyó la información del escrito. El grupo se dividió en equipos que fueron conformados por 5 estudiantes para cada proyecto. Las actividades se calificaron de forma individual y grupal como parte de su calificación final. Se les instruyó para realizar las actividades programadas en la calendarización de la competencia, mismas que se fueron evaluando en el periodo establecido del curso.

Para el tratamiento 3, los estudiantes trabajaron con información obtenida en la competencia para revisar un caso real y realizar un diagnóstico. Dichas actividades fueron realizadas en campo, finalmente fue evaluado.

Finalmente se realizó un cuestionario para obtener información relacionada a los ambientes de aprendizaje, a los cuales estaban expuestos durante el curso. Con el fin de conocer la opinión de cada uno sobre las estrategias de aprendizaje utilizadas por parte del profesor donde se realizaron las actividades.
Para conocer si estos ambientes eran propicios para poder desarrollar sus habilidades al momento de obtener y procesar la información para adquirir el conocimiento de la competencia, plasmarlos en su documento y llévalos en la práctica real.

Para los resultados se realizó un análisis de datos donde se corrió un ANOVA en el programa Excel® para promediar y encontrar las diferencias significativas en las evaluaciones obtenidas por parte de los alumnos en cada una de las subunidades de competencia del curso.

\section{Resultados}

Se obtuvieron en este estudio los siguientes resultados en promedio de cada tratamiento de acuerdo a los ambientes y estrategias de aprendizaje los cuales se muestran a continuación: $\mathrm{T} 1=89 \pm 12.6 .5, \quad \mathrm{~T} 2=78 \pm 14.5$, $\mathrm{T} 3=92 \pm 12.1$, resultando diferentes entre ellos $(\mathrm{P} \leq 0.01)$. El mejor ambiente de aprendizaje se reflejó en el T3, seguido por el T1.

Se encontró que los estudiantes se motivan al estar en diferentes lugares, en los cuales puedan poner en práctica los conocimientos y habilidades adquiridas, además que los ayuda a mejorar su convivencia y participación. Los resultados demostraron que los alumnos prefieren su casa para estudiar, seguido de bibliotecas y cafeterías.

En la encuesta realizada se encontró que el $98 \%$ de los alumnos prefieren ambientes reales para poder practicar lo visto en teoría durante la clase. Mientras que el $2 \%$ le costó trabajo llevar a práctica lo aprendido. Se encontró que la mayoría prefiere desarrollan habilidades con el uso de los recursos y las herramientas virtuales. Con este ambiente ellos pueden administrar sus tiempos y elaborar un documento en Google drive para poder compartir, trabajar en equipo y al mismo tiempo colaborar sin la necesidad de juntarse. Ya que esta aplicación se puede hacer desde su celular o cualquier computadora conectada a internet.

Las encuestas también revelaron que la adquisición de habilidades les proporciona nuevos conocimientos, y coincidieron que la forma virtual de trabajar los motivaba a organizar y administrar el tiempo dedicado al estudio. 
Se encontró que la mayoría de los estudiantes (100\%) tiene acceso a internet, en alguna hora específica del día. Empleando entre 3 y 4 horas buscando información, en redes sociales, en plataformas, para hacer tareas o simplemente para estar de ociosos, perdiendo el tiempo.

\section{Discusión}

El tipo de ambientes de aprendizaje motiva a los estudiantes y al mismo tiempo adquieren las habilidades cognitivas desarrollando principalmente las de tipo social para contribuir y socializar su aprendizaje. Rubí et. al., (2017) mencionan que, el éxito de los ambientes de aprendizaje se debe al papel dinámico que el docente establece cuando interactúa con sus estudiantes logrando así una asistencia diaria a clase logrando un proceso de enseñanza aprendizaje. Por lo que coincide con nuestro trabajo que el buscar los mejores ambientes de aprendizaje motiva y a la vez ayuda a mejorar el proceso de enseñanza-aprendizaje en los estudiantes. Por otra parte los alumnos expuestos a ambientes diferentes desarrollan más capacidades y están más interesados en actividades extra clase.

Herrera y Lorenzo (2009), en su investigación encontraron que el $70 \%$ de los resultados, evidenciaron que el ambiente en que habitualmente suelen estudiar los alumnos influye en sus notas, así como las estrategias de estudio utilizadas, de igual modo los tiempos de dedicación dentro y fuera del aula, son elementos clave para la adquisición de los saberes de la competencia, el estudio coincide con el presente trabajo ya que la aplicación de los diferentes ambientes y estrategias utilizadas denotaron mejores notas en el aprovechamiento. Se vio que el profesor debe de continuar siendo un guía, orientador y facilitador el proceso de aprendizaje, en la mayoría de los estudiantes.

Los resultados obtenidos en este estudio fueron favorables en la realización del proyecto integrador, Cuetia y SanJuán (2017), mencionan que las prácticas en las que se pretenden incluir animales pueden producir un efecto sobre el comportamiento, las emociones y los pensamientos del participante que se beneficia de estas sesiones, tanto en contextos clínicos como educativos.
Lo que concuerda con nuestro estudio donde los estudiantes obtuvieron los mejores resultados al estar en un ambiente real con un caso expuesto al momento de realizar un diagnóstico. En el cual el uso de ambientes reales motivó a los alumnos a que desarrollaran habilidades para complementar su conocimiento. Los resultados obtenidos de los estudiantes mediante el uso de las rubricas aplicadas a las actividades superaron las expectativas esperadas, siendo muy satisfactorio de acuerdo a la calidad de los trabajos entregados por los estudiantes.

Cabe destacar que debe de existir el interés y/o preocupación por parte del docente para aplicar nuevos ambientes de aprendizaje, si estos no funcionan, para cumplir con los objetivos y que las estrategias didácticas utilizadas mediante la aplicación de tecnologías utilizando herramientas y recursos digitales. Los ambientes virtuales permiten crear ambientes de aprendizaje enriquecidos donde los estudiantes pueden visualizar procesos complejos e interactuar con ellos, lo cual puede aportar ciertas ventajas para la realización de trabajos prácticos (Huamani et. al. 2017, Pantoja, et. al. 2017.). Pero sin olvidar la importancia del apoyo, guía y motivación por parte del profesor en la realización de las diferentes actividades del contenido del curso.

Navaridas y Jiménez, (2016) demuestran que el estudio individual y las explicaciones del profesor siguen siendo los ambientes más valorados por los estudiantes para el logro de los objetivos de aprendizaje universitario. Los estudiantes denotan voluntad, responsabilidad e interés para dedicación a su estudio y la importancia del espacio destinado a la adquisición de nuevos conocimientos. El cual debe de ser el adecuado para cada actividad y de esta forma de pueda lograr que se desarrollen las habilidades y competencias para aplicarlas y de esta manera sacar buenas notas y por ende superar las expectativas del docente.

El constante cambio de lugar de trabajo fue una estrategia para que los alumnos salieran de su zona de confort, el cual obligo a que los estudiantes a socializar y compartir el conocimiento adquirido al estar trabajando en equipos $y$ al mismo tiempo utilizar las herramientas virtuales disponibles en nuestros tiempos, permitiendo desarrollar competencias y habilidades para mejorar el desempeño académico.

MEDINA-LERENA, Miriam Susana, COLÍN-MARTINEZ, Marco Antonio, RIMOLDI RENTERÍA, Ma. De Jesús y PÉREZ-RAMÍREZ, Miguel Ángel. Efecto de los ambientes de aprendizaje en estudiantes de manejo de la salud y bienestar animal de la licenciatura de medicina veterinaria en la UdeG. Revista de Pedagogía Crítica. 2019 


\section{Conclusión}

Se concluye que los ambientes de aprendizaje son utilizados por estudiantes y profesores con el fin de adquirir el conocimiento por medio de técnicas y estrategias que garanticen el desarrollo de habilidades y competencias. Sin olvidar que los mejores ambientes de aprendizaje son aquellos que pueden ser usados en la práctica real.

Por otro lado conocer la importancia de la motivación por parte del docente, lo cual representa un factor positivo hacia los estudiantes. Al momento que ellos valoran su evaluación con las actividades y tareas correspondientes en la competencia. En el sentido que constituye una vivencia satisfactoria de retroalimentación como de crecimiento personal y social. En virtud de esto, es indispensable que el docente cambie su forma de percibir y valorar el proceso de enseñanzaaprendizaje.

El objetivo de este estudio se cumplió, ya que los ambientes de aprendizaje utilizados en este estudio ayudaron a buscar que los alumnos demostraran sus habilidades y conocimientos. De igual forma es importante revisar y modificar constantemente los complementos que son utilizados como herramientas para la evaluación de las habilidades y conocimientos de la competencia, de esta manera ayuda a aumentar las probabilidades de éxito en los estudiantes.

\section{Referencia}

Cuetia Londono, J. A., \& SanJuán Sarabia, M. P. (2017). Diseño de un módulo de inducción a las ciencias agropecuarias, mediada por TIC, para los grados décimo y undécimo del colegio Edmundo Velásquez, del corregimiento de Otaré municipio Ocaña norte de Santander, Institución Universitaria Politécnico Gran colombiano, Facultad de Ciencias Sociales, Departamento Académicos de Psicología. Tesis doctoral.

Herrera Torres, L., Lorenzo Quiles, O. (2009). Estrategias de aprendizaje en estudiantes universitarios. Un aporte a la construcción del Espacio Europeo de Educación superior. ISSN 0123-1294. Educación, educación, 12(3):75-78.
Huamani Cruz, R. M., Suni, Y., \& Yesenia, K. (2018). El uso de laboratorios virtuales en la enseñanza del curso de biología y la influencia en el rendimiento escolar de los estudiantes del cuarto grado de educación secundaria de la institución educativa 40616. Arequipa, Perú. Obtenido de http://repositorio.unsa.edu.pe/handle/UNSA/86 65

Maldonado Peréz, M. y Sánchez, T. (2012). Trabajo colaborativo en el aula: Experiencias desde la formación docente. Revista Educare. 16 (2): 93-118.

Márquez P., Prats A. M. (2011). ¿Podemos mejorar con las TIC los resultados académicos? Obtenido dehttp://www.peremarques.net/docs/investigaci onortografia.pdf

Moreno Martínez, M. M. (2005), Un camino para prender a aprender. Guía autodidacta. México, Editorial Trillas, S.A.

Navaridas Nalda, F. y Jiménez Trens, M. A. (2016). Concepciones de los estudiantes sobre la eficacia de los ambientes de aprendizaje universitarios. Revista de Investigación Educativa, 34(2), 503-519. Obtenido de http://dx.doi.org/10.6018/rie.34.2.239481

Pantoja, C., \& Carlos, J. (2017). Diseño de una multimedia interactiva en el aprendizaje de ciencias naturales sobre el efecto invernadero en las y los estudiantes del décimo año de educación básica, de la Unidad Educativa José Joaquín Olmedo, de la parroquia Olmedo, Cantón Cayambe, 2016 (Bachelor's thesis, Quito: UCE). Obtenido de http://www.dspace.uce.edu.ec/handle/25000/12 710.

Rodríguez Vite, H. (2014). Ambientes de Aprendizaje. Universidad Autónoma del Estado de Hidalgo. Boletín Científico, Ciencia Huasteca. Vol. 2, No. 4, ISSN: 2007-493X Obtenido dehttps://www.uaeh.edu.mx/scige/boletin/hueju tla/n4/e1.html 
Rubí Sánchez, G. O., Vásquez, J. D. L. Á., \& Rueda, X. E. (2017). Ambientes de aprendizajes y su incidencia en el proceso enseñanza aprendizaje de los Estudiantes del Segundo Grado de la Escuela Pública Unión Centroamericana del distrito VI de Managua durante el segundo semestre del año 2017. Tesis, Universidad Nacional Autónoma de Nicaragua, Managua. Obtenido de. http://repositorio.unan.edu.ni/8641/

Saraiva, L.M., Pernigotti J.M., Barcia R.M., Lapolli E.M. (2006). Tensionsthataffectdistancelearningsettings.

PsicoloEstudo. 11(3):483-91

Villamizar Acevedo, G. (2008). Relación entre estrategias de aprendizaje y rendimiento académico de estudiantes de psicología. Revista Docencia Universitaria. 6(9): 71-94. 